
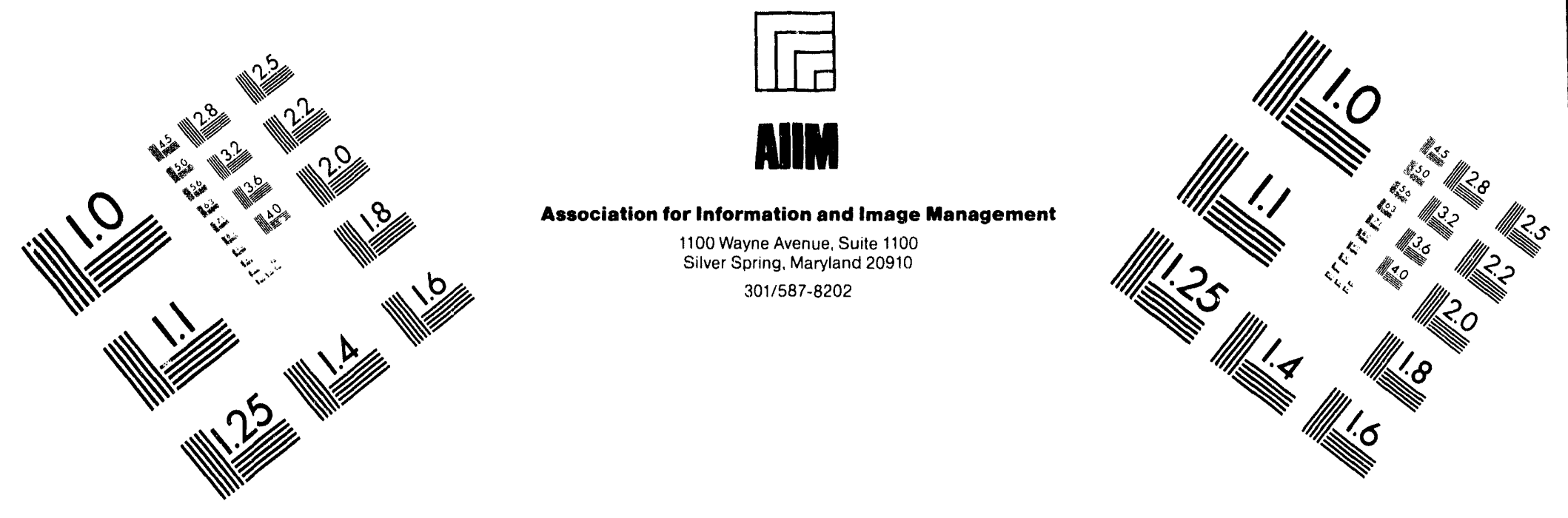

Centimeter

$\begin{array}{llllllllllllllll}1 & 2 & 3 & 4 & 5 & 6 & 7 & 8 & 9 & 10 & 11 & 12 & 13 & 14 & 15 & \mathrm{~mm}\end{array}$

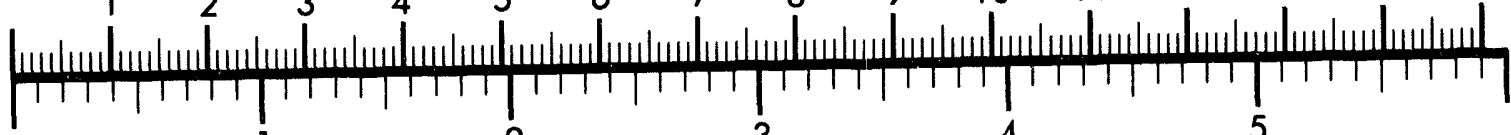
Inches

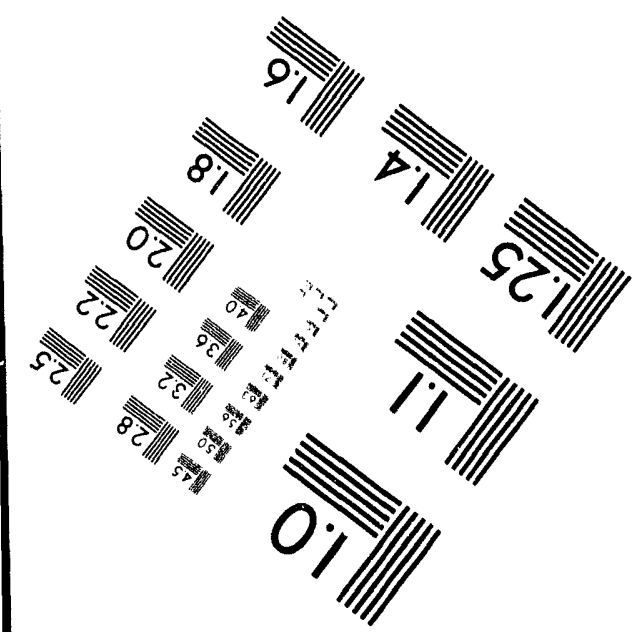

MANUFACTURED TO AIIM STANDARDS

BY APPLIED IMAGE, INC.

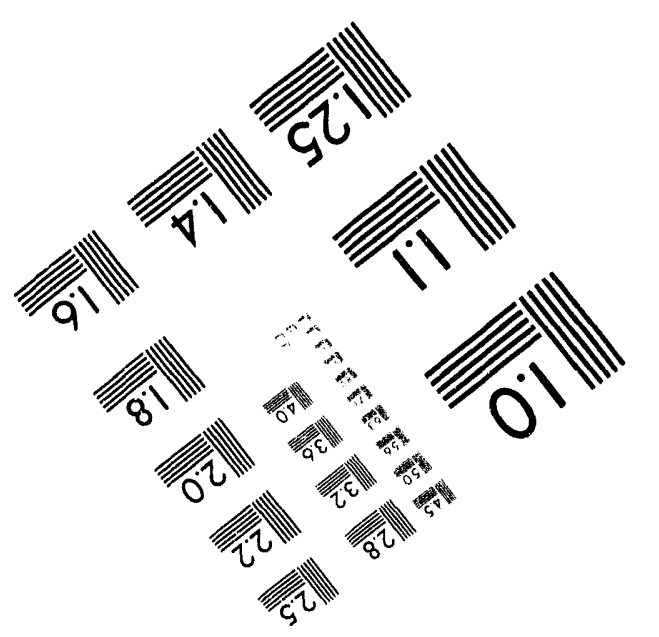



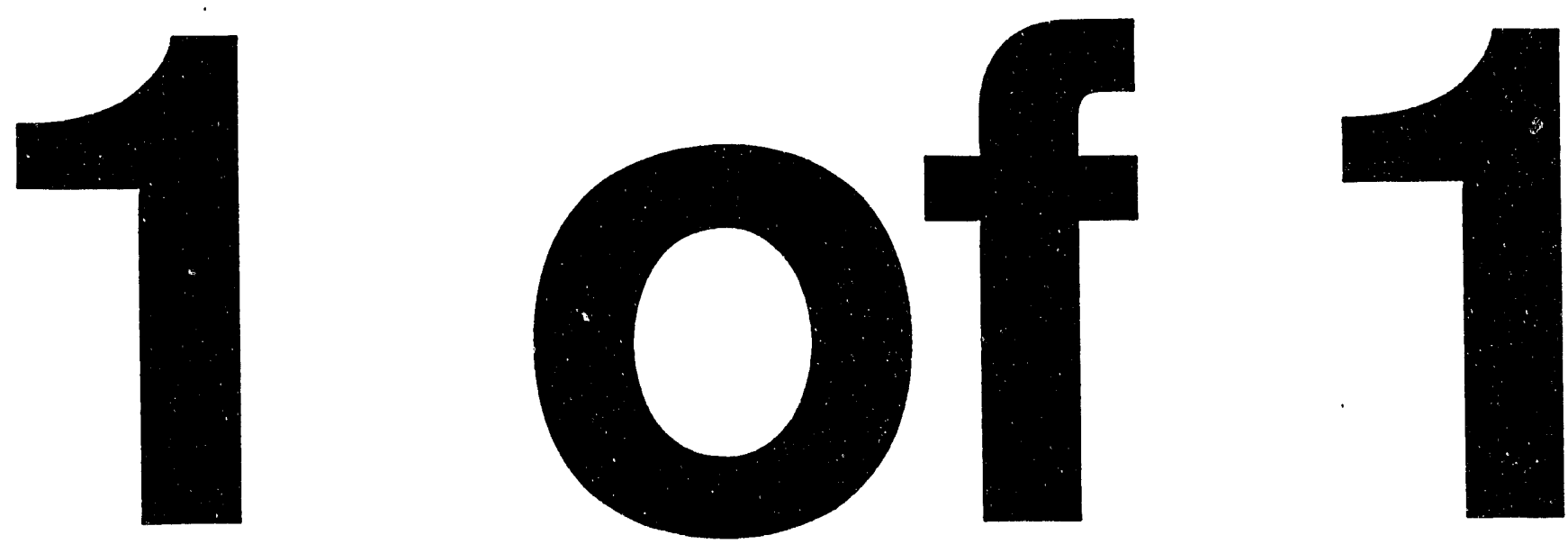


\title{
APPLICATION OF ARTIFICIAL INTELLIGENCE TO RESERVOIR CHARACTERIZATION: AN INTERDISCIPLINARY APPROACH
}

(DOE Contract No. DE-AC22-93BC14894)

\author{
Submitted by
}

The University of Tulsa

Tulsa, OK 74104

\author{
Contract Date: \\ October 1, 1993 \\ Anticipated Completion Date: September 30, 1996 \\ Government Award: \\ Program Manager: \\ B.G. Kelkar \\ Principal Investigators: \\ R.F. Gamble \\ D.R. Kerr \\ L.G. Thompson \\ S. Shenoi \\ Reporting Period: \\ January 1 - March 31, 1994 \\ Contracting Officer's Representative \\ Mr. Robert E. Lemmon \\ Pittsburgh Energy Technology Center \\ P.O. Box 10940, M/S 141-L \\ Pittsburgh, PA 15236-0940
}

\begin{abstract}
DISCLAIMER
This report was prepared as an account of work sponsored by an agency of the United States Government. Neither the United States Government nor any agency thereof, nor any of their employees, makes any warranty, express or implied, or assumes any legal liability or responsibility for the accuracy, completeness, or usefulness of any information, apparatus, product, or process disclosed, or represents that its use would not infringe privately owned rights. Reference herein to any specific comnercial product, process, or service by trade name, trademark, manufacturer, or otherwise does not necessarily constitute or imply its endorsement, recommendation, or favoring by the United States Government or any agency thereof. The views and opinions of authors expressed herein do not necessarily state or reflect those of the United States Government or any agency thereof.
\end{abstract}
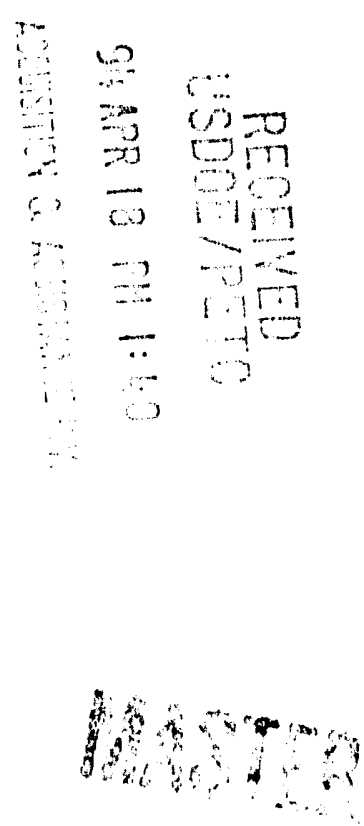


\section{Objectives}

This basis of this research is to apply novel techniques from Artificial Intelligence and Expert Systems in capturing, integrating and articulating key knowledge from geology, geostatistics, and petroleum engineering to develop accurate descriptions of petroleum reservoirs. The ultimate goal is to design and implement a single powerful expert system for use by small producers and independents to efficiently exploit reservoirs.

The main challenge of the proposed research is to automate the generation of detailed reservoir descriptions honoring all the available "soft" and "hard" data that ranges from qualitative and semi-quantitative geological interpretations to numeric data obtained from cores, well tests, well logs and production statistics. In this sense, the proposed research project is truly multi-disciplinary. It involves significant amount of information exchange between researchers in geology, geostatistics, and petroleum engineering. Computer science (and artificial intelligence) provides the means to effectively acquire, integrate and automate the key expertise in the various disciplines in a reservoir characterization expert system. Additional challenges are the verification and validation of the expert system, since much of the interpretation of the experts is based on extended experience in reservoir characterization.

The overall project plan to design the system to create integrated reservoir descriptions begins by initially developing an AI-based methodology for producing largescale reservoir descriptions generated interactively from geology and well test data. Parallel to this task is a second task that develops an AI-based methodology that uses facies-biased information to generate small-scale descriptions of reservoir properties such as permeability and porosity. The third task involves consolidation and integration of the large-scale and small-scale methodologies to produce reservoir descriptions honoring all the available data. The final task will be technology transfer. With this plan, we have carefully allocated and sequenced the activities involved in each of the casks to promote concurrent progress towards the research objectives. Moreover, the project duties are divided among the faculty member participants. Graduate students will work in teams with faculty members.

The results of the integration are not merely limited to obtaining better characterizations of individual reservoirs. They have the potential to significantly impact and advance the discipline of reservoir characterization itself. 


\section{Summary of 'Technical Progress}

\section{Decomposition of System}

We have decomposed the overall system development into smaller component parts to allow us to focus on the expert knowledge required for that component. In addition, the decomposition will facilitate the implementation of the system and its validation and verification. The three component systems will be representative of how each of the experts in geology, geostatistics, and engineering characterizes the reservoir. Figure 1 describes a model for this breakdown. The concurrent development of these component systems fits into the development of the large and small scale aspects of the system as originally stated in the proposal. In Figure 1, each component system in the model is depicted as interfacing (through the bi-directional links) with a central repository of reservoir descriptions. Tìough, portions of these description will essentially be passed from component to component as more information is gathered (as shown by the bidirectional links in Figure 2), the model of a central repository is an accurate account of how the components are integrated, i.e., the final descriptions in the repository are consistent will all of the information given by the components systems. This system model allows us to develop the system using an Artificial Intelligence technique called a blackboard system, in which information is centrally located, i.e., on a blackboard, and experts take their turn to update, change, and correct the information on the blackboard.

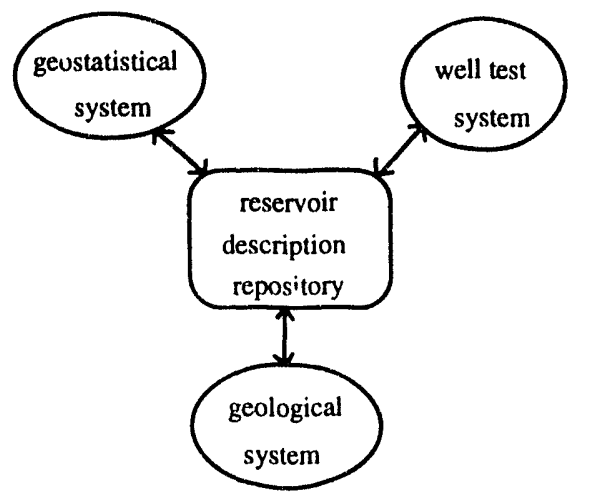

Figure 1: Expert System Decomposition

We have begun the develop of a small prototype in Kappa-PC to gain an understanding of the representation issues in order to provide each system with the information that it needs, either as raw input or as feedback information from another system.

Figure 2 presents a more detailed decomposition of Figure 1 and includes the current raw input information that we are currently examining (as designated by the double arrows). The bi-directional links indicate where feedback will occur between systems, when more information is learned and inconsistencies in descriptions are 
discovered. In the remaining sections, we present the knowledge acquired at this time and the development stage of the individual component systems.

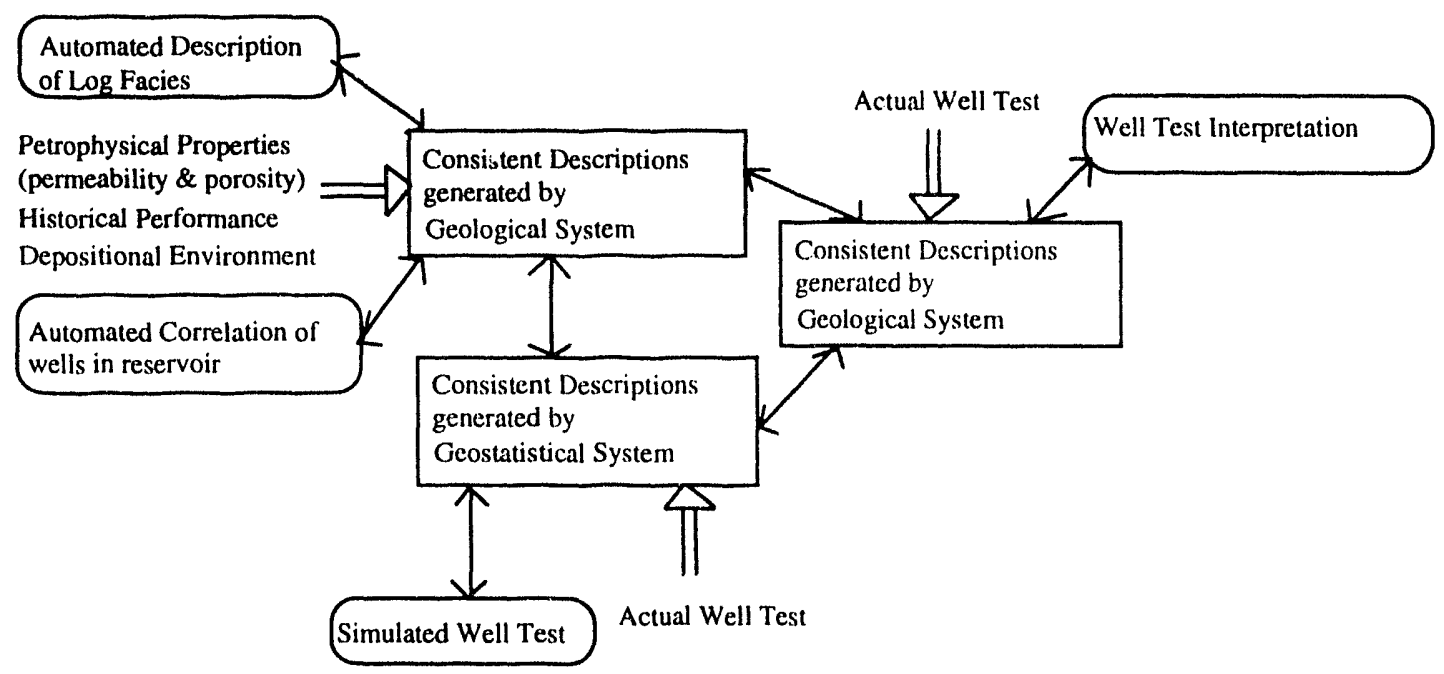

Figure 2: Detailed Decomposition of the System

\section{Geostatistical System: Incorporating Dynamic Constraints in Description Process}

Stochastic methods have gained widespread acceptance as a promising methodology for enhancing our ability to describe the spatial distribution of reservoir properties. Conditional simulation, because of its ability to honor the sample data and create multiple equiprobable reservoir descriptions if required, has been selected as the stochastic method which will be use for this study. Specifically, the conditional simulation algorithm to be employed is the Simulated Annealing Method. ${ }^{1}$ This method was selected because of its robustness and the facility and flexibility of incorporating conditioning data.

Traditionally, only static constraints, such as core and log data, have been employed in the simulated annealing process. While the inclusion of dynamic constraints, such as well test and production data, is considered desirable, practical considerations prevented their use in large scale projects. One major impediment has been the required computational for using a dynamic constraint.

The primary objective of this work is to develop a methodology for incorporating dynamic constraints, such as well test and production data, into the simulated annealing algorithm. Improved reservoir description can be obtained for the following reasons:

i. We minimize the uncertainties in describing reservoirs by adding more constraints. In addition, the descriptı ' $n$ will also be closer to the "truth case" as we add more information. This implies that our description approaches a closer approximation of the reservoir being studied.

ii. Dynamic constraints, e.g. production and pressure data, provide the only observable response that is representative of reservoir-scale behavior, along with 
providing "in-situ" information. Other available data, including log and core data, represent reservoir data which is limited to the near-wellbore region.

iii. We c.ln eventually combine this work with geological constraints to create a truly integrated reservoir description.

Our approach involves the coupling of a numerical flow simulator to the simulated annealing algorithm. The flow simulator constitutes a dynamic constraint as part of the simulated annealing objective function, that consist of a variogram constraint and a flow simulation constraint.

Simulated annealing is an example of adaptive heuristics for multivariate or combinatorial optimization. The implementation requires, among other parameters, the definition of an objective function that represents a measure of the difference between an input model and our "fit" of that model. Thus, the goal of the algorithm is to determine the configuration of independent variables -- for example, permeability values -- that minimize the objective function. When the objective function is comprised of more than one model, we attempt to minimize the sum of the differences.

The Laplace Transform Finite Difference (LTFD) method is used for the flow simulation. This method is based on solving the single-phase flow equations in Laplace space. It has the advantage of using only one timestep between the initial time and the time of interest. As a result, this method is far less computationally expensive than other numerical methods.

The annealing method has been modified so that the perturbations of the permeability field are no longer random but radiate outwards from the well location(s). This modification was performed after we observed that, especially in early times, the near-wellbore conditions had a significant effect on the production response.

The first phase involved testing the performance of the LTFD numerical simulator against a commercial simulator (ECLIPSE was used). The results of this successful test are shown in Figure 3. This figure shows a comparison of the output pressures for a heterogeneous reservoir from ECLIPSE and the LTFD simulator at different times for a two-well system with each well producing at a variable rate. 
Comparison of LTFD \& ECLIPSE Output Pressures

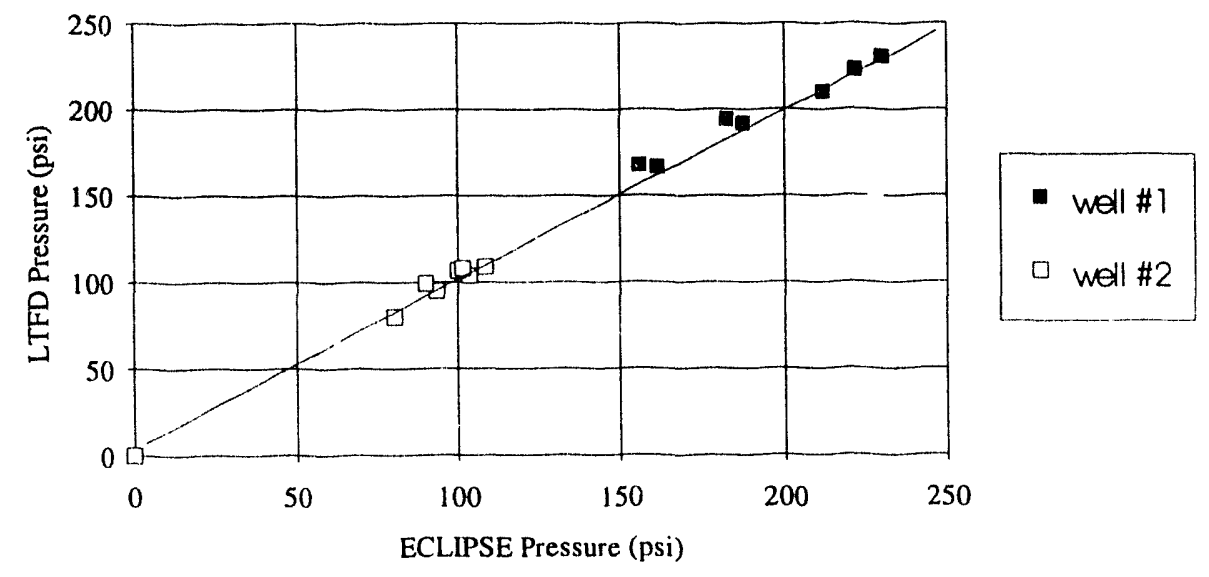

Figure 3

In the second phase, a synthetic permeability distribution was generated and used to test the performance of the algorithm. Conditioning data (permeability values) at two well locations and a cumulative distribution function representing the synthetic input data were included in the input constraints.

One of the tests executed compared the production behavior of the reservoir model obtained by using both constraints to the results with only the variogram constraint. The results showed a dramatic improvement (see Figures 4 and 5) in the agreement with the synthetic input data when the simulator cunstraint was included.

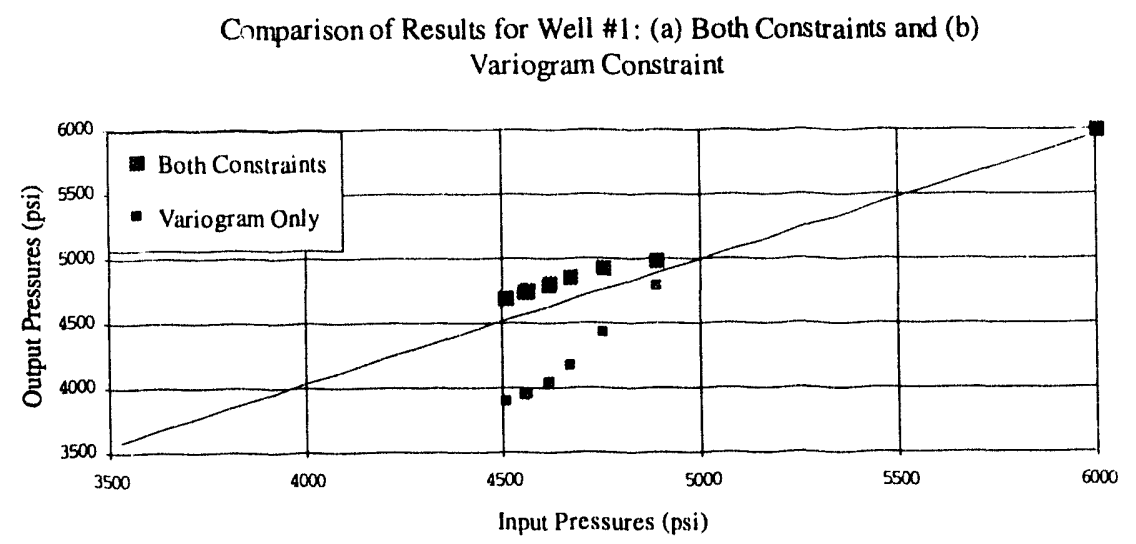

Figure 4 
Comparison of Results for Well \#2: (a) Both Constraints and (b) Variogram Constraint

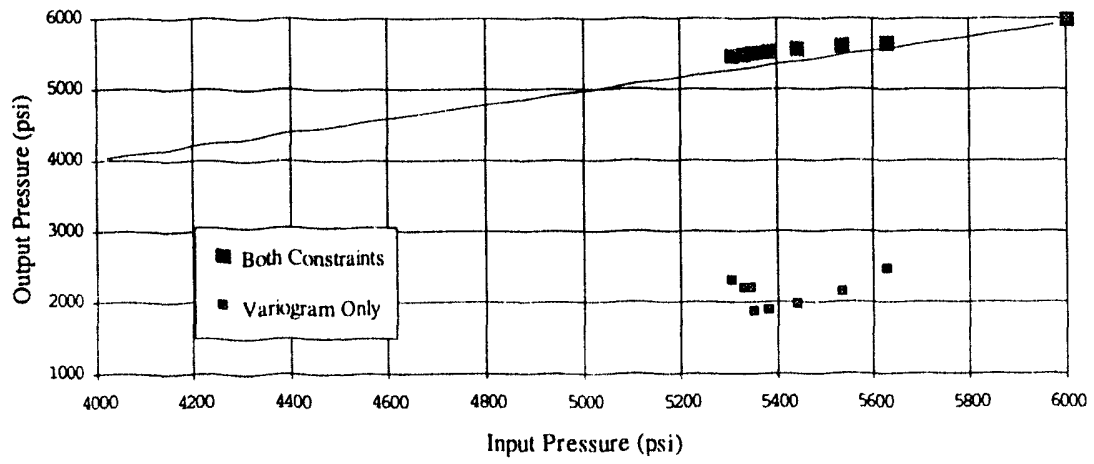

Figure 5

While the results are encouraging, the algorithm remains computationally demanding. Additional work is needed to included the effect of convergence tolerances on the final solution. We need to further optimize the matrix solving routines, or sourcing of better routines. In addition, the code needs to be streamlined and optimized. Eventually, we would like to integrate this program with other routines to include known geological constraints.

\section{Well Test Interpretation System}

In addition to the above research, we are also taking a more traditional expert system approach by developing a well test interpretation. The development of such a system involved gathering knowledge from petroleum engineering experts to determine the appropriate model and apply the associated equations of that model. Transient testing is a major source of vital information about reservoir parameters like permeability, reservoir pressure, wellbore conditions, reservoir discontinuities and other information that is essential for reservoir studies. The accuracy of these properties estimated from well tests depends on prior identification of a model that describes the reservoir accurately. This model is known as the "well test interpretation model".

The procedure for finding an appropriate model can be quite complex. It usually resides deep in the expert's mind. The failure of mathematical models to solve the problem can be attributed to the nature of the problem itself since the procedure is not completely quantitative and relies on experience.

Computerized well test involves interpreting the various forms of the time versus pressure data, in addition to other well data, to determine the well model and calculate the various parameters. Basically, this interpretation can be grouped into two parts: qualitative analysis and the quantitative analysis. Qualitative analysis deals with selecting the appropriate well model from the input data. Quantitative analysis involves calculating various well parameters like permeability, skin factor (if applicable) etc. The estimation 
of well properties depends upon the selection of the right model, making qualitative analysis a crucial part of the problem.

The expert system we are developing for well test interpretation consists of rules and facts for buildup test analysis. The initial rules simulate the reasoning process used by the experts to identify the appropriate interpretation model for a well test. Within this approach, the expert system is designed to use a description of the shapes of different graphs of test data, particularly the derivative plot. When needed, the system seeks information in addition to test data from relevant sources, such as known reservoir and fluid properties, production statistics, well logs and geological data.

The expert system architecture consists of three main components: a knowledge base consisting of facts and rules, a database consisting of supplied data and model parameters, and a control strategy to determine when to use the expert rules in the knowledge base. Although knowledge is the most important part of the knowledge base, the control problem is a critical component in the design of the system. Simulating the human expert's reasoning process requires control strategy to organize the reasoning steps and domain knowledge to achieve an efficient way of constructing a solution to a problem. Figure 6 describes the flow of information through the modules of the system.

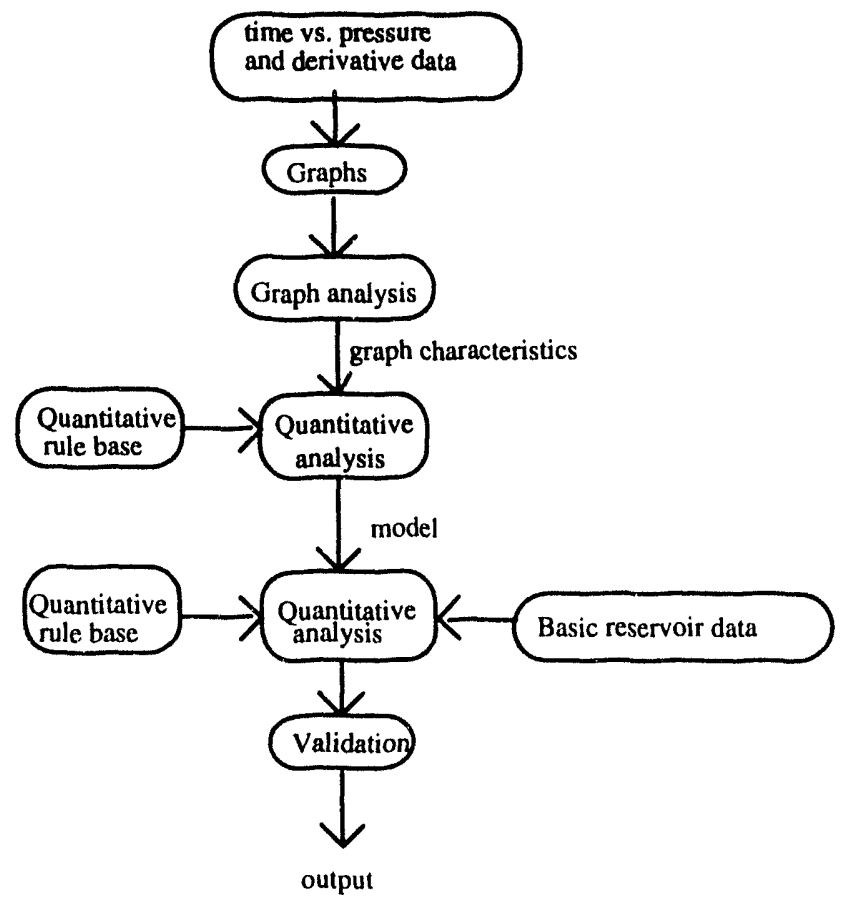

Figure 6: Well Test Expert System Model

The above diagram shows the different modules in the system. The first module takes in the time vs. pressures data and the derivative data. In the absence of the derivative data, this module may be used to calculate the derivative data from the existing time and pressure data. The graph drawing module is used to plot the graph. This module is required to apply smoothing techniques and eliminate the noisy data. The sketch algorithm presented in 1988 by Allain and Horne ${ }^{3}$ extracts a set of consecutive 
log-log segments from a potentially noisy derivative. A hybrid neural network based scheme was presented by Allain and Houze 5 . The algorithm chosen for this module should be able to draw a smooth plot and eliminate noisy data. Though the system concentrates on the derivative plot, other plots may be required. This is especially true since the derivative plot is more sensitive to noise. The other plots used to confirm the results include the semi-log plot, log-log plot and the Horner plot.

The graph analysis module would take the graph plotted in the previous step, identify flow regions and look for typical characteristics in the graph. The derivative plot is very well suited for this purpose because it intensifies the response of each flow regime. There are basically a limited number of signatures on the derivative curve that can be attributed to each flow region. These signatures include maxima, minima, stabilization (flattening), and upward and downward trend. Shape recognition algorithms are used for this purpose.

The signatures identified in the above module are given to the qualitative analysis module. This module is responsible for the model selection based on the flow regions and the corresponding signatures. This module would have to access the qualitative rule base which consists of rules for selecting the models based on the plot trends. In case of the absence of a flow region (incomplete data) or the inability to select a model based on the incomplete graph data, this module would utilize the available geological data. In addition, the user can provide a preference over model selection.

The quantitative analysis module is used to calculate the reservoir parameters once the model is selected. This module would have to access a rule base containing the equations used by different models. It would also need basic reservoir data supplied by the user.

The verification module is used to verify the deduced model. This module may also be used to select between models when more than one model is obtained. The verification method would use the interpretation model reservoir parameters (e.g., permeability, skin, etc.) as initial estimates in an automatic history matching algorithm. ${ }^{4}$

The initial input to the system consists of pressure vs. time data, basic fluid properties (compressibility, viscosity, density) and basic rock properties (porosity, fluid saturations). This input is used by the system to generate the diagnostic plots. Later in the process, the system will seek other relevant data when needed.

In a complete well test analysis, the expert often uses diverse information sources to find the applicable interpretation model. These knowledge sources are shown in Figure 7. 


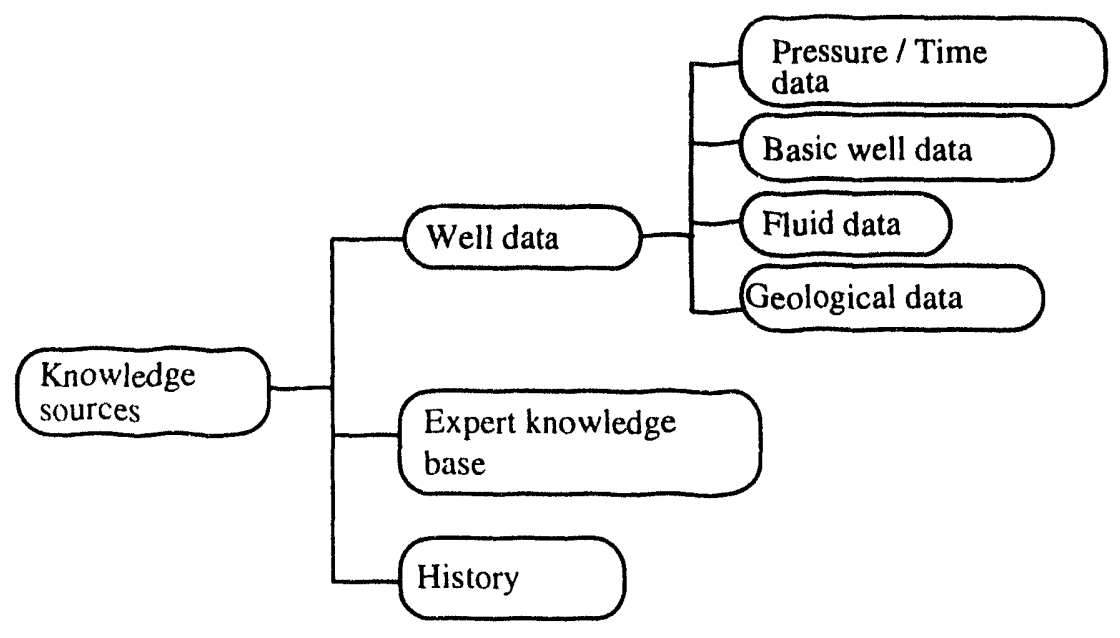

Figure 7: Expert System Knowledge Sources

The first category shown in Figure 7 is the data related to the response of the physical properties of the well and the reservoir. These include pressure and flow data, well data, fluid data and field data (which includes interpreted geological data) The second category is the expertise or the expert's knowledge. This category forms the critical part of the knowledge base because it affects the whole reasoning process. The third category contains mathematical models and other data to simulate the known reservoir behavior. This category is used to validate and confirm the interpretation obtained from the knowledge base.

The model recognition process will be carried out by first dividing the plot into flow regions. The well test interpretation model can be partitioned into three components: the reservoir behavior, the behavior at the inner boundaries, and the behavior at the outer boundaries. Each of these is associated with a well-defined flow region in the well test data. These regions are the early-time, middle-time, and the latetime regions. The early-time region is associated with the wellbore and near-well conditions; the middle-time region is associated with the reservoir behavior; and the late time region is associated with the outer boundary conditions.

The system would identify the time intervals spanned by the flow regions before attempting to identify the interpretation model segments. Figure 8 shows the different regions and the different conditions and behavior recognized by the system in each region. 


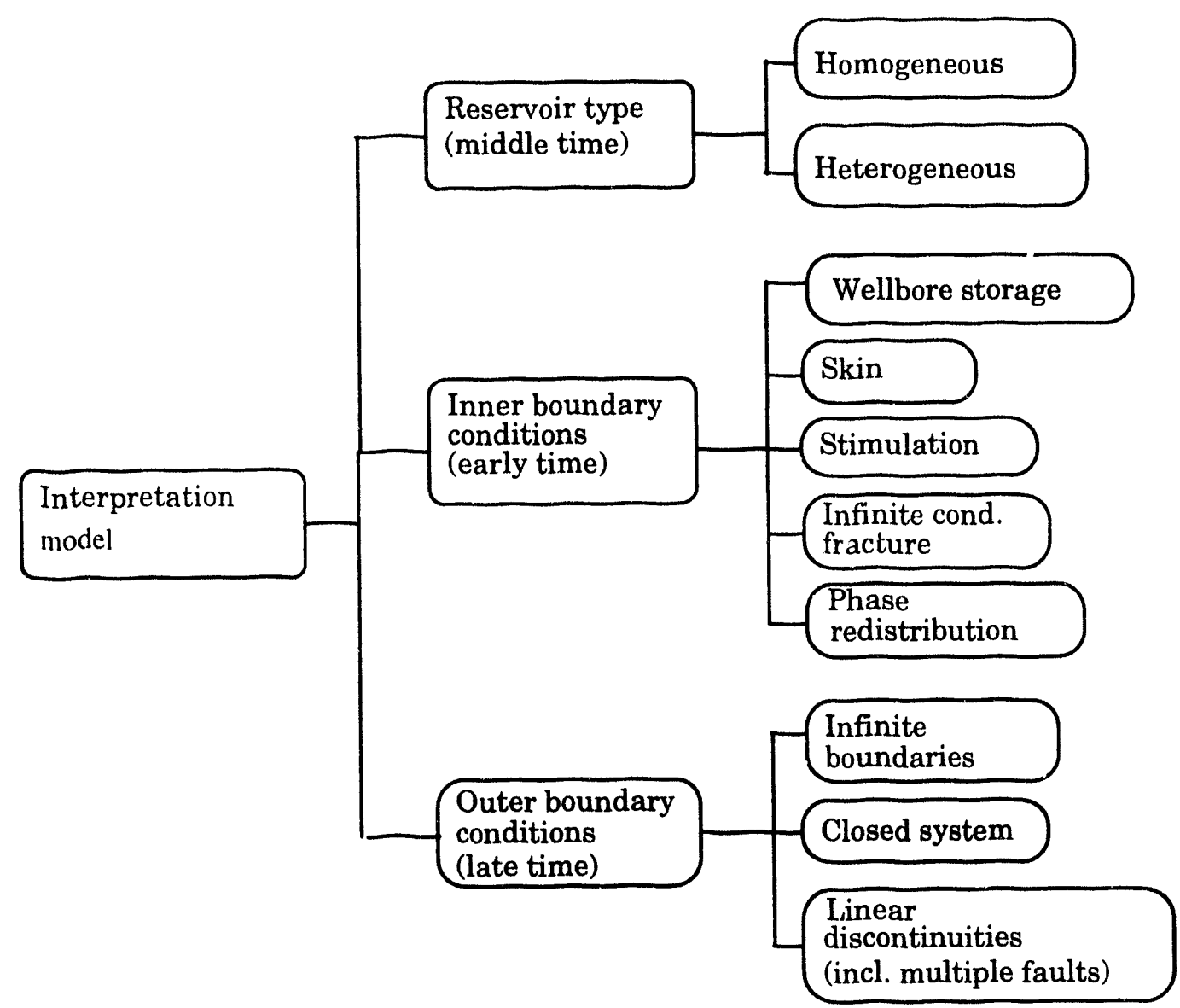

\section{Figure 8: Conditions and Behavior Recognized by each Region}

We use the available expert's knowledge for separating the flow regimes and interpreting the responses of the flow regimes on the derivative curve. The change from one flow regime (behavior) to another produces a distinct change in the shape of the curve. The procedure, based on the left-to-right visual inspection of the derivative curve, will be fully automated. The system would start by selecting a marker point (e.g., a point of inflection). The recognition process then proceeds by expanding around the marker and describing the characteristic shapes that precede or succeed the marker zone. User interaction may be taken at this stage to select the marker if required. The process ends when all the regions have been defined. It is possible that the system will conclude that one or more regions are missing, a situation that is arises because of incomplete well data.

Determination of the interpretation model will begin with defining the reservoir type (middle-time region) to see whether the reservoir is homogeneous or heterogeneous. Once the reservoir type is defined, the system will proceed to check the early-time region of the graph, where the system determines the inner boundary conditions. The system will be able to distinguish the following inner boundary conditions: wellbore storage, skin, stimulation, infinite conductivity fracture, finite conductivity fracture, and phase redistribution. The well can show any logical combinations of the above conditions. The last segment the system would resolve is the late-time region. The system will be able to differentiate between three outer boundary conditions: an infinite acting reservoir, a 
completely closed reservoir, and linear discontinuities. The system will allow any logical combinations of these outer boundary conditions.

It is possible that at the end of the session, one or more segments are not resolved because of incomplete data or inconsistent conclusions are reached because of incorrect data. In this case, the system will seek help from information sources other than the test data such as well reports, geological information, etc. It is also possible that it will identify more than one possible solution for a segment of the interpretation model. In such cases, both of the solutions will be considered for the rest of the analysis. Final selection will be based on the verification process or the user's choice. The output given by the system is the final selected model and the calculated reservoir properties like permeability, skin, etc.

Identification of the interpretation model is accomplished by focusing on individual flow regions. The system starts by identifying the reservoir model using the middle region characteristıcs of the derivative plot. The rules required in this step are those that determine if the reservoir model in homogeneous or heterogeneous. A typical rule for identifying the reservoir model is as follows:

If The derivative plot has a minimum

And Minimum is V-shaped

And The semi-log plot shows two parallel straight lines

Or The semi-log plot shows a flat line (transition) followed by a straight line

And The log-log plot shows a slope change

Then The reservoir model is heterogeneous

The log-log plot and the semi-log plot are used to confirm the observations from the derivative plot. The middle time region of the plot without a minimum indicates a homogeneous model. Trends in the plot may sometimes indicate the possibility of two different models.

If Minimum is observed in the middle time region of the derivative plot

And Minimum has open U-shape

And Semi-log plot shows evidence of two straight lines with possible slope doubling

And Log-log plot indicates slope change

Then The reservoir model is heterogeneous

Or The reservoir model is homogeneous with possible linear discontinuity (fault)

The above rule leads to the identification of two models: heterogeneous model and homogeneous model with linear discontinuity. Both the models produce similar test data plots.

After identifying the reservoir model, the system then looks at the early-time region of the plot. Rules in this step identify wellbore storage, skin, stimulation, infinite conductivity fracture, phase redistribution, etc. The following trends suggest typical inner boundary conditions: 
If Plot shows a unit slope line

And Plot shows a hump

Then Indicates presence of wellbore storage

If Plot has a 1/4 slope line

Then Presence of finite conductivity fracture

If Plot has a $1 / 2$ slope line

Then Presence of infinite conductivity fracture

The above trends may be confirmed by looking at the semi-log and the log-log plot. After identifying the inner boundary conditions, the system will look at the late-time region in the graph to identify the outer boundary conditions. This includes identifying infinite boundary conditions, closes systems and linear discontinuities (including multiple faults). Typical rules in this step are as follows:

If The derivative plot is horizontal

And Semi-log plot is a single stright line

And The log-log plot shows one line (no inflection point)

Then The reservoir is infinite acting

If The derivative plot shows an upward increase followed by stabilization

And The upward increase is preceded by stabilization

And Evidence of second straight line on the semi-log plot with doubling of slope

Then Linear discontinuity exists in a homogeneous system

Or A heterogeneous system is infinite acting

In cases where more than interpretation is possible, geological information and well test results from wells in the same area may provide further information and help in selecting a model. Model verification through history matching also can confirm a particular selected model.

\section{Geological System}

This system is further decomposed into two subsystems as depicted in Figure 2. We have implemented some of the functionality of the system in Kappa-PC in an effort to determine a frame-based representation of the system and its information. Screen printouts of the Kappa-PC program are presented in Figure 10 at the end of the report.

\section{Log Facies Description System}

The initial development of the log facies description system concentrates only on gamma ray logs. We are examining and artificial log, at first, to determine the location of sand bodies and then drawing the corresponding logs. Our initial experiments have been limited to five different wells. The drawings were then digitized and stored in Microsoft Excel. We use Excel to automate the normalization of the data, as shown by 
the 0 to 100 scale on the $x$-axis of the graphs below, and correlate the data (a process previously done by hand using Excel). Once implemented in Kappa-PC, the system will use specific functions to directly interact with Excel to utilized the previously derived information. The expert system portion will include rules similar to the following rule to classify the $\log$ facies.

Symmetrical Rule

If $\quad\left(\mathrm{D}_{1} 10-\mathrm{D}_{\mathrm{u}} 901\right)=\left(\mathrm{D}_{\mathrm{l}} 90-\mathrm{D}_{\mathrm{u}} 101\right)$ Then "symmetrical"

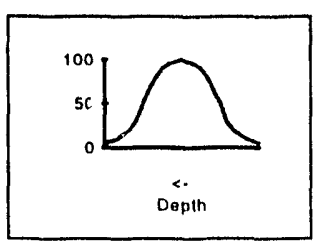

If the absolute value of the lower Depth of the curve at $\mathbf{1 0}\left(D_{1} 10\right)$ minus the upper Depth of the curve at $90\left(D_{u} 90\right)$ is equal to the absolute value of the lower Depth of the curve at $90\left(D_{1} 90\right)$ minus the upper Depth of the curve at $10\left(D_{u} 10\right)$, then the curve is symmetrical.

Bell Rule

$$
\text { If } \quad\left(\left|D_{1} 10-D_{1} 90\right|\right)<\left(D_{u} 90-D_{1} 10 \mid\right)
$$

Then "bell"

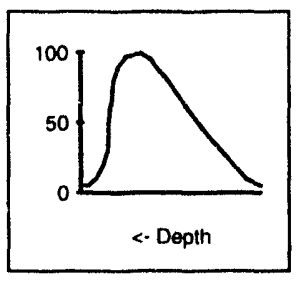

If the absolute value of the lower Depth of the curve at $\mathbf{1 0}\left(D_{1} 10\right)$ minus the lower Depth of the curve at $90\left(D_{1} 90\right)$ is less than the absolute value of the upper Depth of the curve at $90\left(D_{u} 90\right)$ minus the upper Depth of the curve at $10\left(D_{1} 10\right)$, then the curve is bell shaped.

Funnel Rule

$$
\begin{aligned}
& \text { If } \quad\left(\left|D_{1} 10-D_{l} 90\right|\right)>\left(\left|D_{u} 90-D_{u} 10\right|\right) \\
& \text { Then "funnel" }
\end{aligned}
$$

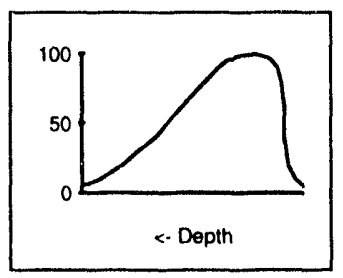

If the absolute value of the lower Depth of the curve at $10\left(D_{1} 10\right)$ minus the lower Depth of the curve at $90\left(D_{1} 90\right)$ is greater than the absolute value of the upper Depth of the curve at $90\left(D_{u} 90\right)$ minus the upper Depth of the curve at $10\left(D_{u} 10\right)$, then curve is funnel-shaped.

Blocky Rule

$$
\begin{aligned}
& \text { If } \quad\left(\left|D_{1} 10-D_{1} 90\right|\right)==\left(\left|D_{u} 90-D_{u} 10\right|\right) \\
& \text { Then "blocky" }
\end{aligned}
$$

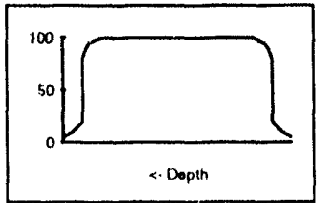


If the absolute value of the lower Depth of the curve at $\mathbf{1 0}\left(D_{1} 10\right)$ minus the lower Depth of the curve at $90\left(D_{1} 90\right)$ is equal to the absolute value of the upper Depth of the curve at $90\left(D_{u} 90\right)$ minus the upper Depth of the curve at $10\left(D_{u} 10\right)$, then the curve is blocky.

\section{Correlation System}

Once the log facies for each well have been classified, the information in the wells must be correlated across the reservoir. The initial development of this module of the expert system has begun by identifying specific expert rules for correlation, and then determining an appropriate representation of the reservoir information within Kappa-PC. Stratigraphic correlation means the determination of equivalent depositional units prevalent throughout a reservoir under consideration. The types of equivalency include lithostratigraphic, referring to similarities in sedimentary rock types using lithologic criteria; biostratigraphic, referring to similarities in fossil content; and chronostratigraphic, referring to similarities in the time of deposition. The wireline log data are used extensively to determine the equivalency between various stratigraphic units.

Given that $d t 1$ and $d t 2$ are the depths from the marker bed to the top of the first sand in wells 1 and 2, respectively, we present some very basic rules for well to well correlation.

If $\mathrm{dt} 1=\mathrm{dt} 2$ or nearly so

Then probably the units marked 1 in the two wells are correlatable.

If the $\log$ facies of both units are similar

Then it is even more likely that the two stratigraphic units are correlatable.

If the log facies of both units are not correlatable

Then the two units are discontinuous in the middle and they are not stratigraphically equivalent. Move to the next units in sequence.

The above rules are translated into a Kappa-PC function called divide as represented below. 
AppendToList(WellGroupl:ListOfWells,i);

Else \{AppendToList(WellGroup2:ListOfWells,i);\};\};\});

This function operates on our initial frame-based representation of the reservoir. A frame-based representation is used in artificial intelligence system to combine the advantages of object-oriented system with rule-based reasoning techniques. Kappa-PC offers such a frame-based representation. The representation of the reservoir is presented below in Figure 9.

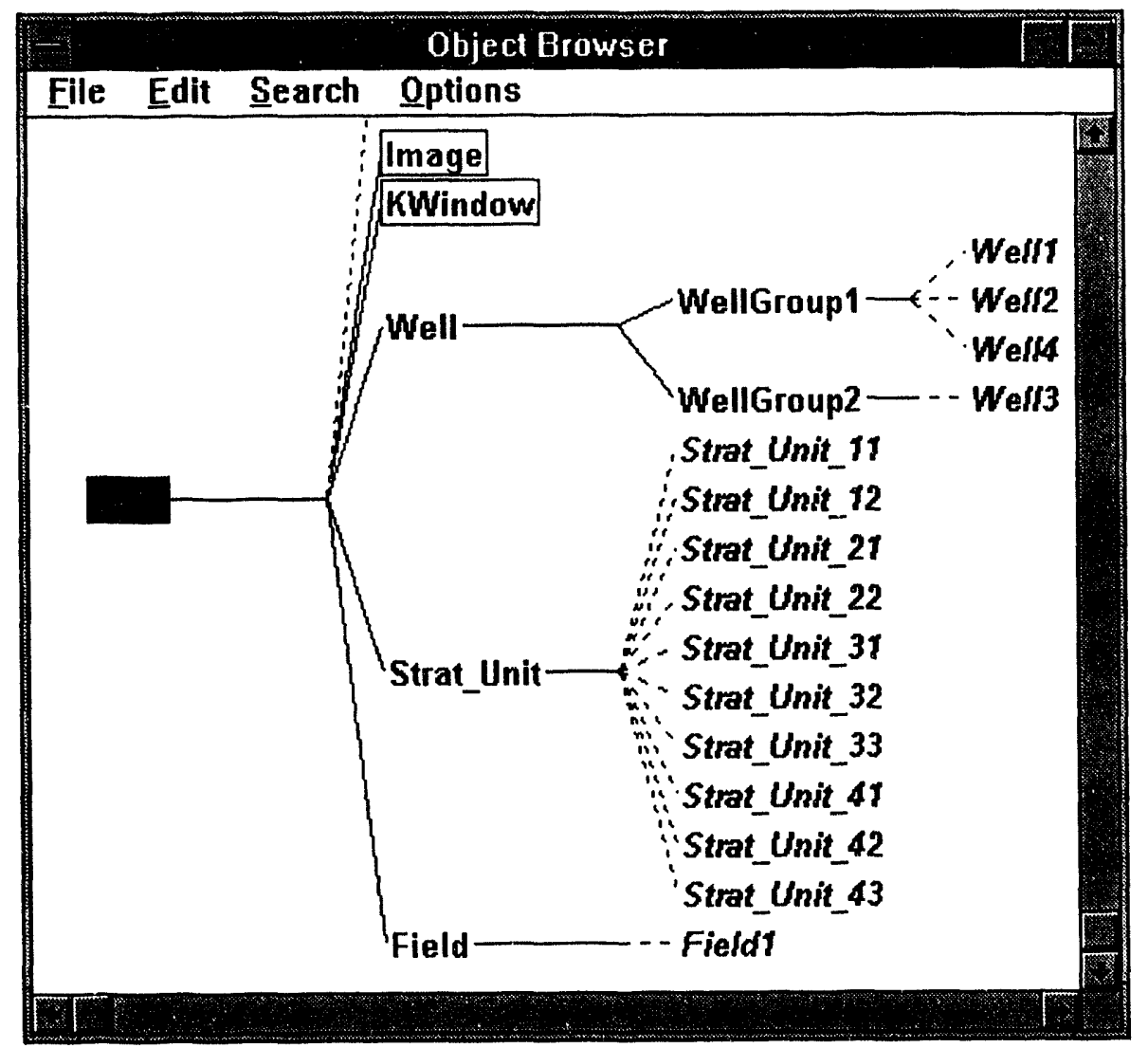

Figure 9: Frame-Based Representation of Reservoir

In Figure 9, the class unit are in regular font. There is a class of wells, stratigraphic units, and oil fields. The information in italicized font are the instances of the class. For example, in the figure, there are four well instances. The stratigraphic unit instances can be recognized as being numbered (by the last number) within a well (by the first number). At this time in the computation, the divide function has been executed to separate the wells into correlatable (WellGroup1) and undetermined (WellGroup2).

Once it is determined that the wells are possible correlatable, the next step is to find the correlation coefficient between the two units. A high correlation coefficient would indicate that they are correlatable. The correlation coefficient should be computed taking into account the expansion index between the two units under comparison. 
In addition, the expert system tries to match other evidences such as drill cutting analysis, side-wall core analysis, fossil content, etc. The more closely the parameters are related, the more the system increases its certainty in the stratigraphic equivalency.

The following rules pick the next stratigraphic units in the two wells and repeat the above procedures.

If $\mathrm{dt} 1$ is less than $\mathrm{dt} 2$

Then either the 1st sand on well1 is a pinch out or it is thinning towards well2.

If there is strong evidence of similarities between log features, drill cuttings, sidewall cores or fossil contents

Then probably unit 1 of well 1 is thinning towards well 2

Else it is probably a pinch out.

If unit 1 of well 1 is a pinch out

Then correlate second unit with the first of well 2.

If unit 1 of well 1 is thinning towards well 2

Then move to the second stratigraphic units on both the wells and start correlation.

If $\mathrm{dt} 1$ is greater than $\mathrm{dt} 2$

Then either the 1st sand on well 2 is a pinch out or it is thinning towards well 1.

If there is strong evidence of similarities between log features, drill cuttings, sidewall cores or fossil contents

Then probably unit 1 of well 2 is thinning towards well 1 .

Else it is probably a pinch out.

If unit 1 of well 2 is a pinch out

Then correlate second unit with the first of well 1 .

If unit 1 of well 2 is thinning towards well 2

Then move to the second stratigraphic units on both the wells and start correlation.

The Kappa-PC function correlate below illustrates a portion of the correlation represented by the above rules. 


\section{REFERENCES}

1. Perez, Godofredo: "Stochastic Conditional Simulation for Description of Reservoir Properties", PhD Dissertation, The University of Tulsa, Tulsa, OK (1991)

2. Kirkpatrick, S., Gelatt Jr., C.D. and Vecchi, M.P.: "Optimization by Simulated Annealing", Science (May 13 1983) pp 671-680.

3. llain, O. F. and Horne, R. N.: "Use of Artificial Intelligence in Well-Test Interpretation", JPT (March 1990) pp. 342 - 349.

4. Watson, A. T. and Lee, W. J.: "A New Algorithm For Automatic History Matching Production Data ", paper SPE15228, 1986

5. Houze, O. P. and Allain, O. F.: "A Hybrid Artificial Intelligence Approach in Well Test Interpretation", paper SPE24733, 1992. 

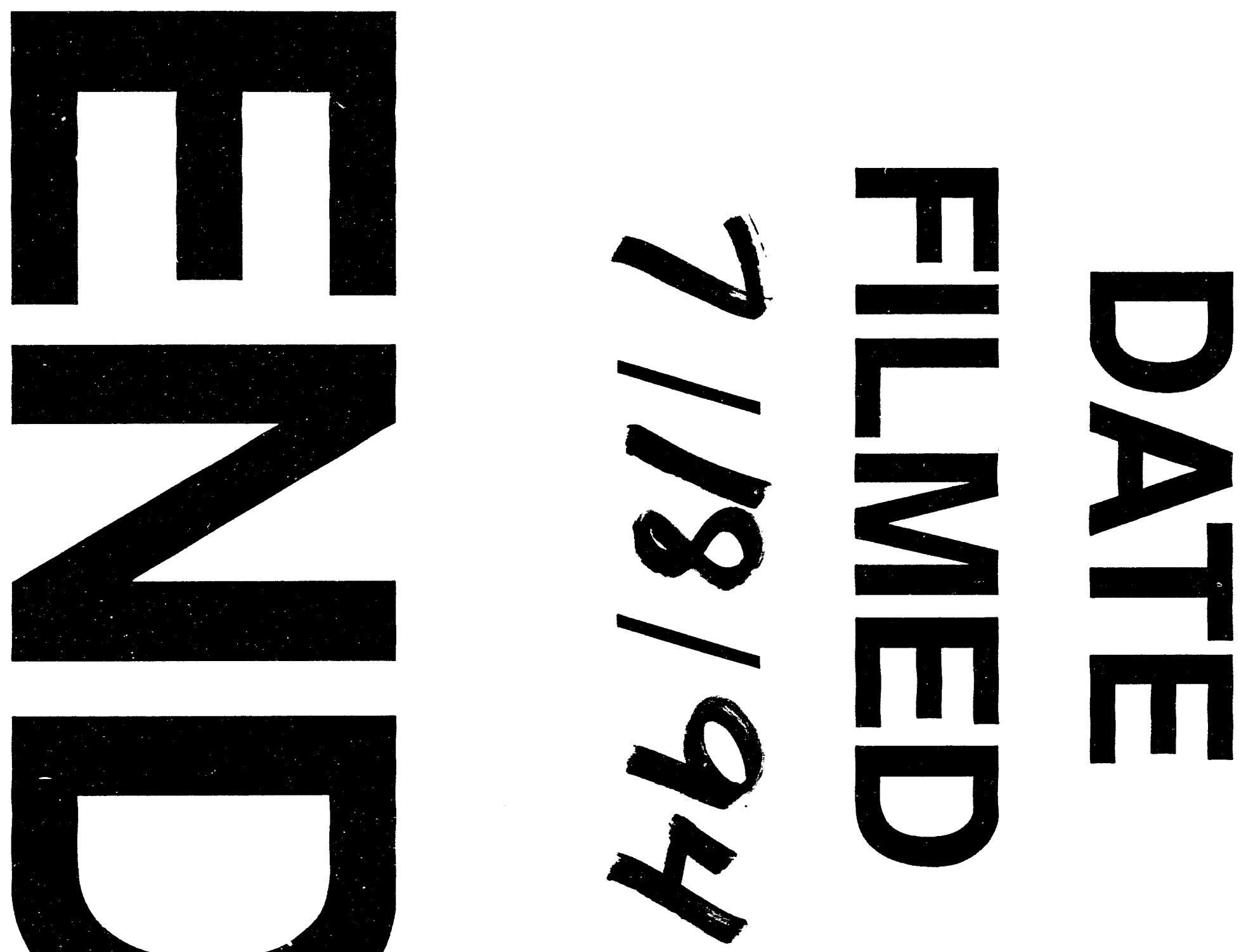

$\theta$

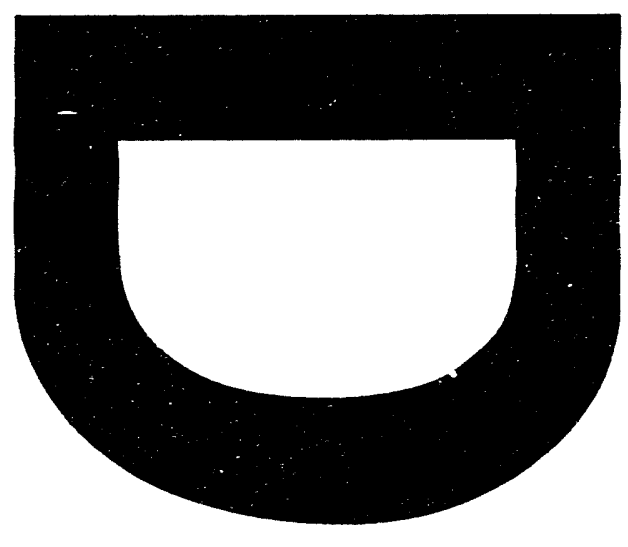


